\title{
La expansión Uruk y el proceso de urbanización: una perspectiva desde la Alta Mesopotamia
}

\section{Leandro Constanze Lima \\ Universidad de Buenos Aires, Argentina}

Fecha de recepción: 25 de julio de 2021. Fecha de aceptación: 18 de agosto de 2021.

\begin{abstract}
Resumen
En la región de la Alta Mesopotamia, durante el período que abarca el Calcolítico Tardío 4 y 5 (3600-3000 a.C.) se observa la aparición de diversos asentamientos con características urbanas. El surgimiento de estos sitios y el material arqueológico hallado en los mismos planteó diversas cuestiones respecto a su origen. En este artículo proponemos un relevamiento del acervo teórico, haciendo foco en los modelos que relacionan a estos centros urbanos con una dinámica de centro-periferia y el análisis desde la perspectiva de las redes de intercambio regionales, sin dejar de lado el desarrollo propio interno de cada asentamiento. Consideramos particularmente tres sitios específicos que presentan vertientes distintas de formación urbana donde la intensidad de la injerencia externa también es variable. En el yacimiento de Habuba Kabira observamos una fuerte presencia de población Uruk, lo que podría responder al asentamiento de una diáspora dominante proveniente de la Baja Mesopotamia. Mientras que en el sitio de Hacinebi Tepe parecen convivir una comunidad local y una comunidad foránea autónoma inserta en ella. Finalmente, en Tell Brak se manifiesta un centro autóctono independiente que, según nuestro parecer, se habría apropiado de ciertos rasgos culturales Uruk.
\end{abstract}

Palabras clave: Alta Mesopotamia, urbanismo, Calcolítico tardío, expansión Uruk

The Uruk Expansion and the Urbanization Process: A Perspective from Upper Mesopotamia

\author{
Abstract \\ In the Upper Mesopotamia region, during the period encompassing the \\ Late Chalcolithic 4 and 5 (3600-3000 BC) various settlements with urban
}


characteristics appear. The emergence of these sites and the archaeological material found in them raised diverse questions regarding their origin. In this paper a focus is placed on the models that link these urban centers with a centerperiphery dynamics and analysis from the perspective of regional exchange networks, without leaving aside the internal development of each settlement. Three specific sites are considered, with different aspects of urban formation and highly variable intensity of external interference. At the Habuba Kabira site we observed a strong presence of Uruk population, which could respond to the settlement of a dominant diaspora from Lower Mesopotamia. At the Hacinebi Tepe site, however, the Uruk population seemed to live together a local community and an autonomous foreign community inserted in it. Finally, Tell Brak had an independent indigenous center that could have made an appropriation of certain Uruk cultural traits.

Keywords: Upper Mesopotamia, urbanism, Late Calcolithic, Uruk expansion

\section{Introducción}

Durante la segunda mitad del IV milenio a.C. se consolidaron las primeras ciudades en la Alta Mesopotamia, varias de las cuales parece que tuvieron un fuerte contacto con otras urbes de la Baja Mesopotamia, particularmente Uruk, moderna localidad de Warka (Adams y Nissen, 1972; Liverani, 2006 [1998]). El objetivo de este trabajo es establecer las características de estas interacciones y la influencia que pudieron haber tenido en el proceso de urbanización. Para esto focalizamos nuestra atención en tres regiones, seleccionando un sitio característico en cada una de ellas: Éufrates medio (Habuba Kabira), Éufrates alto (sitio de Hacinebi Tepe) y triángulo del Khabur (sitio de Tell Brak). Nuestro recorte temporal se basa en las fases 4 y 5 del período Calcolítico Tardío (de ahora en más CT), datadas aproximadamente entre 3600 y 3000 a.C. Estas últimas corresponden al llamado fenómeno Uruk, cuando la cultura material originaria de la Baja Mesopotamia se expandió hacia la Alta Mesopotamia y otras regiones del Cercano Oriente (Algaze, 1989; 2004; Stein, 1999; Schwartz, 2001; Postgate, 2002). De todas formas, realizaremos breves referencias a las fases inmediatamente anteriores, pues entendemos que no podemos comenzar partiendo del instante en que aparecen los primeros restos de Uruk, ya que de esta manera obtendríamos una visión sesgada.

Pero, antes de llevar adelante esta pesquisa, pasamos revista a los modelos teóricos que se han postulado hasta el momento para explicar los vínculos entre las poblaciones locales y la Baja Mesopotamia. Cuando observamos el proceso de urbanización en las regiones en cuestión, la formación de estos asentamientos se presenta heterogénea. La fundación de los mismos no responde a una regla general respecto al momento de su aparición, a las características de su construcción, al material cultural hallado o a su devenir histórico. En este sentido, es especialmente difícil determinar el origen preciso del proceso de urbanización.

Los tres asentamientos urbanos que tomamos como referencia se encuentran a considerable distancia entre sí y de Uruk; sin embargo, todos presentan en algún momento cierto contacto con la Baja Mesopotamia y compartieron una 
característica: tuvieron un rol territorial estratégico en sus regiones respectivas y en la comunicación con otras zonas más alejadas. Cuando hablamos de la evidencia material, hacemos foco en tres tipos de restos: la arquitectura, la cerámica y la glíptica. Esta evidencia nos permitió establecer comparaciones con aquellos materiales característicos de la cultura Uruk. Aun así, también tomamos en menor medida otros restos hallados para profundizar en la investigación. Tal es el caso de yacimientos de extracción de bitumen y despojos animales dentro de los asentamientos.

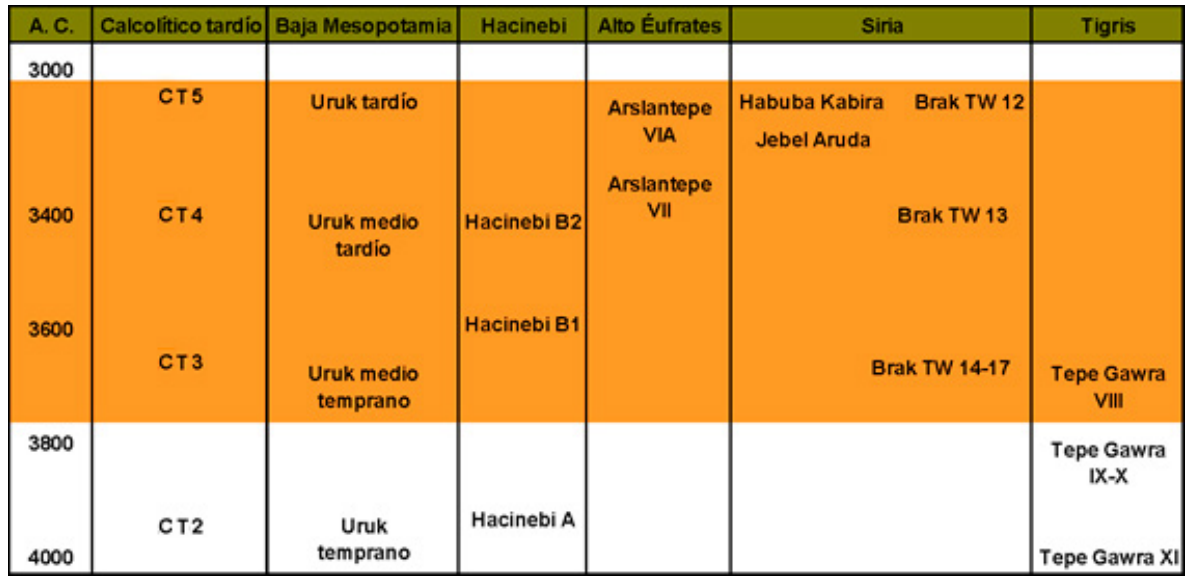

Tabla cronológica de los sitios durante el IV milenio a.C. (elaborada siguiendo a Schwartz y Hollander, 2008).

A partir de los resultados obtenidos, trabajamos con las teorías que en las últimas décadas han profundizado sobre las interacciones en la región. El variado escenario teórico nos permitió comparar ciertos modelos que definen el tipo de relación que habría desarrollado Uruk con estos sitios, así como aportar nuevas perspectivas integradoras, retomando la evidencia disponible y con el objetivo de brindar mayor luz sobre los contactos regionales durante la segunda mitad del IV milenio a.C.

\section{Modelos teóricos}

A continuación, abordamos sintéticamente cinco modelos teóricos que se han utilizado para analizar el contacto cultural en relación con el fenómeno Uruk: el modelo del sistema-mundo, el modelo de aculturación, el modelo distance-parity, el modelo de la diáspora de comercio y el modelo de desarrollo regional alternativo. El primero "sugiere que el crecimiento económico de las sociedades complejas puede ser mejor entendido observando las redes interregionales de intercambio a gran escala, compuestas por varias entidades políticas competidoras" (Stein, 2002: 904). La formulación clásica de este modelo proviene de Immanuel Wallerstein (1974), que en principio no lo pensó para las sociedades antiguas, sino para el surgimiento del moderno sistema capitalista mundial. Su aplicación para el contexto del Cercano Oriente antiguo fue esbozada por Kohl (1987) y luego reafirmada entre otros por Algaze (1989; 2004 [1993]). 


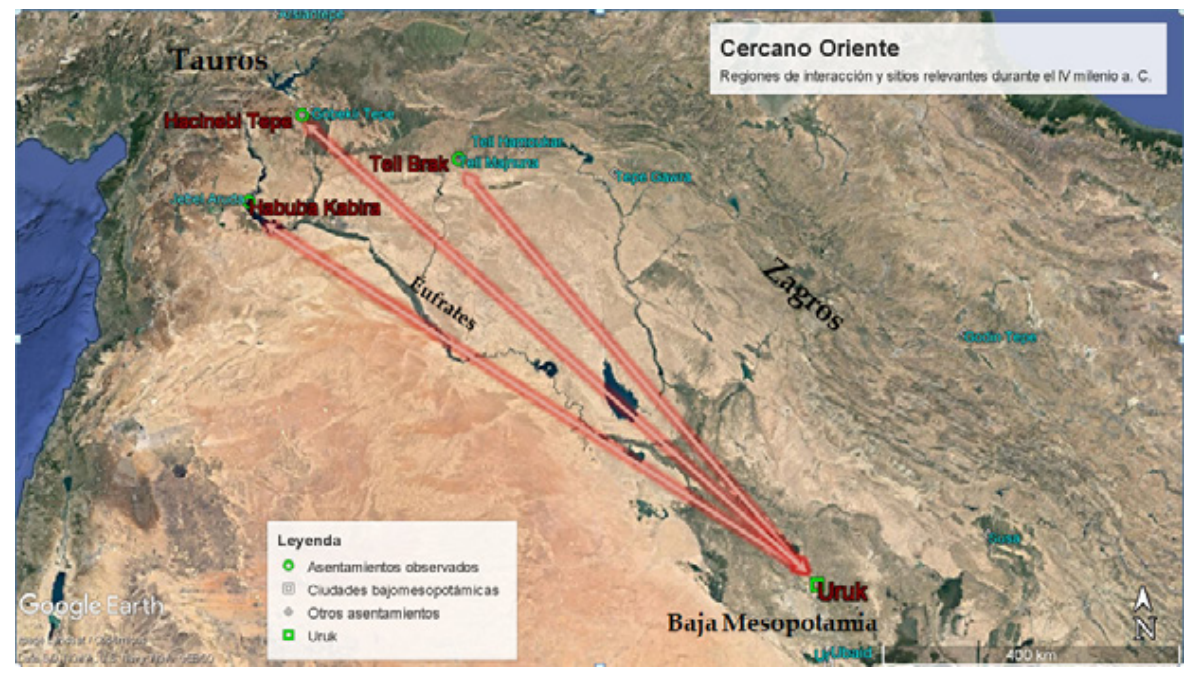

Mapa del Cercano Oriente con los sitios mencionados en el artículo (realizado con Google Earth).

La premisa principal del modelo de sistema-mundo es la existencia de dos polos opuestos: centro y periferia. Partiendo de aquí, la teoría se caracteriza por la dominación del centro, el establecimiento de un intercambio asimétrico entre el centro y la periferia, y la primacía causal de la interacción de larga distancia a la hora de estructurar la economía política en la periferia. El foco de la teoría está puesto en el factor externo que actúa sobre las sociedades periféricas, siendo éste el elemento fundamental en su formación económica y social.

El modelo de la aculturación, por su parte, también presenta en su primera elaboración una separación binaria entre culturas recipientes y sociedades donantes. Los grupos recipientes, más débiles y menos desarrollados, progresivamente van tomando las características de las sociedades donantes que los controlan. Este proceso ocurre a través del préstamo de diferentes trazos culturales y lleva inevitablemente a la desaparición del grupo pequeño, integrado por la cultura más amplia (cf. Juliano, 1988). Sin embargo, esta interpretación se centra en la asimilación, cuando el proceso de aculturación también se ha entendido desde un proceso reciprocitario entre culturas. En los últimos años, autores como Butterlin (2003) han trabajado en esta perspectiva, considerando la expansión a través no de la invasión sino del contacto cultural de larga duración en las regiones involucradas. En sus trabajos sobre la glíptica regional en el período Calcolítico, Pittman (2001) también ha retomado la influencia del contacto Uruk en el intercambio cultural con el norte de la Mesopotamia.

Volviendo a la teoría del sistema-mundo, cuando Algaze (1989; 2004 [1993]) la aplica al fenómeno Uruk, considera que ciertas condiciones geográficas y ambientales en la llanura aluvial mesopotámica generaron un desarrollo distinto al de otras sociedades en su periferia. La necesidad de Uruk de conseguir materias primas que no se encontraban en su región, condicionó a sus habitantes a entrar en contacto con zonas alejadas que pudieran proveerles de estos bienes. Las relaciones con estas sociedades estuvieron signadas por el interés en obtener madera, piedra, metales y en algunos casos esclavos. La interacción habría sido asimétrica, ya que las sociedades periféricas no se encontraban en el mismo 
nivel de integración social y política que las urbes de la Baja Mesopotamia. A cambio de las materias primas, Uruk posiblemente proporcionaba tejidos y otros objetos elaborados. Su interés era establecer una red de asentamientos para controlar las rutas comerciales y supervisar el flujo de mercancías. Algaze clasificaría de la siguiente forma los tipos de asentamientos:

1) Enclaves Uruk. Se encontraban normalmente en las principales rutas terrestres y cursos de agua. Se trataba de asentamientos centrales de proporciones urbanas rodeados por varios centros satélites menores.

2) Estaciones o bases Uruk. Asentamientos más pequeños, en una posición aislada alejada de los enclaves de la primera categoría. Su función sería la de actuar como enlaces o "estaciones" entre los enclaves y la llanura aluvial mesopotámica.

3) Puestos avanzados. Pequeños asentamientos de tamaño similar a las estaciones o bases, pero aislados en medio de comunidades indígenas. Su función era controlar el flujo de bienes destinados a Mesopotamia.

4) Sitios locales con material Uruk. Numerosos sitios que estarían involucrados directamente en el comercio de materias primas.

Según Algaze, el estado habría sido quien trataba de garantizar la obtención de recursos. Enclaves como el de Habuba Kabira, por ejemplo, se habría creado de forma intencionada directamente por alguna ciudad bajo-mesopotámica. El estado controlaría el intercambio de bienes, fundamentalmente con la exportación de textiles, así como productos acabados. Tendría además un rol fundamental en el almacenamiento y redistribución de materias primas -madera, metales, piedras- en su área de influencia. Algaze no descarta la existencia de un comercio privado controlado por individuos o funcionarios del estado que se valdrían de su posición para el lucro personal. Sin embargo, el intercambio interregional estaría principalmente dominado por el sector público. Por último, el autor plantea que en la región aluvial mesopotámica habría diversos núcleos centralizados de poder compitiendo entre sí. En este marco de enfrentamiento, las ciudades-estado buscarían establecer asentamientos en su periferia para controlar las vías de comunicación y el acceso a recursos foráneos.

De manera simultánea, Mark Schwartz (1988) también había propuesto un acercamiento similar, donde la expansión Uruk estaría ligada al comercio, pero además debido a la necesidad de aumentar los terrenos de explotación agrícola. El contacto entre Uruk y los asentamientos de su periferia habría producido cambios importantes en estos últimos, debidos principalmente a que estas sociedades tendrían un grado de complejidad política menor. No obstante, rápidamente criticó el énfasis que Algaze ponía en la búsqueda de materias primas, ya que esto dejaba de lado otras variables posibles. En este sentido, se pregunta por qué esta suerte de colonización habría sido necesaria, cuando simplemente se podría haber permitido que las comunidades indígenas continuaran con su extracción, lo cual sería mucho más fácil y barato. Aun así, este autor propuso la siguiente clasificación:

1) Colonias Uruk. Asentamientos con una cultura material casi exclusivamente propia de la Baja Mesopotamia y un rol importante en el control del comercio. En esta categoría incluía sitios como Habuba Kabira y Jebel Aruda. 
2) Asentamientos Uruk dentro de un sitio local: Establecimientos comerciales similares al karum paleoasirio. Ejemplos de este tipo serían el yacimiento oval amurallado de Godin Tepe y, tal vez, Tell Brak.

3) Sitios locales en las proximidades de un enclave comercial Uruk. Estos lugares serían reconocibles por una cultura local con algún material intrusivo del sur como sería el caso de Kurban Höyük.

4) Grandes centros locales en contacto con Mesopotamia. Arslantepe VI A sería el mejor ejemplo de asentamientos de este tipo.

5) Sitios locales sin contactos con Mesopotamia.

Posteriormente, Schwartz (2001) redujo su acercamiento a partir de sólo dos tipos de asentamientos:

1) Colonias Uruk: implican la formación de asentamiento en terreno virgen, con una cultura plenamente bajo-mesopotámica visible en la arquitectura, la cerámica, sellos cilíndricos y bullae.

2) Asentamientos locales con presencia de materiales Uruk: sitios en los que se aprecia una cultura local y otra Uruk.

En los años siguientes a la aplicación del sistema-mundo por parte de Algaze, Gil Stein $(1990 ; 2002)$ matizó esta teoría planteando un modelo de distanceparity en el cual el poder hegemónico que puede ejercer el centro va decayendo con la distancia. En este sentido, para el autor (Stein, 2012) las relaciones de poder entre la Baja Mesopotamia y los centros con los que realiza intercambios variaban dependiendo de múltiples factores, donde la distancia sería el rasgo fundamental. En principio, Uruk podría dominar o establecer relaciones asimétricas en el área más cercana, pero a medida que aumentara la distancia con el sur las relaciones de intercambio se tornarían más balanceadas. Así, por ejemplo, la población de un enclave Uruk en Hacinebi Tepe se encontraría en condiciones de igualdad o incluso de desventaja frente a la comunidad local.

Stein formuló además otra alternativa al sistema-mundo que para nosotros resulta fundamental a la hora de pensar las interacciones a larga distancia. Se trata del modelo de diásporas de comercio (Stein, 1999) que hace foco en los participantes de las redes de intercambio. En este sentido, las diásporas se forman en un espacio social étnicamente heterogéneo, el intercambio se realiza frente a grupos culturales distintos, en condiciones de transporte y comunicación complicadas, donde no existen instituciones estatales que puedan proveer seguridad en el intercambio de larga distancia. La diáspora se organiza como grupo cerrado y cohesivo para sobrevivir y prosperar en sus relaciones con la comunidad local y con otros grupos que participen en el intercambio. Nuestro análisis discurre cerca de esta propuesta, por esto será retomada en las conclusiones.

El sentido primordial que Algaze le ha otorgado al intercambio de larga distancia en la expansión del modelo mesopotámico-meridional y su cultura durante el IV milenio a.C. también ha sido discutido recientemente por Marcella Frangipane (2018: 47-48). Según la autora, no hay evidencia de que el intercambio haya tenido un rol prioritario para los estados prístinos durante este período. No discute la existencia de relaciones de comercio interregionales, pero 
considera que su economía estaría enfocada en la centralización de alimentos y de aquellos bienes necesarios para su producción. A nuestro modo de ver, esta autora establece un modelo general de desarrollo local alternativo, donde cada una de las partes se basa en sus propios recursos y formas de organización. En función de lo anterior, Frangipane (2009: 34-35) ha planteado una serie de modelos específicos de estructuración social y territorial para tres regiones distintas:

1) El modelo periférico. Particular de las zonas más alejadas al aluvión mesopotámico, desde el Éufrates alto en los Tauros hasta el este del Tigris. Aquí encontramos centros administrativos que controlaban la agricultura en sus alrededores, así como la extracción de recursos y el intercambio con otras regiones distantes. El urbanismo es poco y en algunos casos nulo, la influencia Uruk se limita a objetos sueltos provenientes del comercio o a pequeños puestos donde habitaría una diáspora débil y dependiente de la autoridad local. Arslantepe, Tepe Gawra y Hacinebi Tepe son ejemplos de este tipo de asentamientos.

2) El modelo del valle del Khabur. Aquí se desarrollaron centros urbanos como Tell Brak o Tell Hamoukar, gracias en gran parte a la productividad agrícola de la región. Estos tuvieron una importante interacción con la Baja Mesopotamia y eventualmente asimilaron sus patrones culturales.

3) El modelo del valle del medio Éufrates. En la zona del Éufrates sirio y el sureste de Anatolia la penetración Uruk fue más determinante y directa, tal es el caso de Habuba Kabira y Jebel Aruda. Esto ocurrió debido a la falta de una autoridad dominante previa en el área.

Ya en años recientes, Algaze (2004 [1993]) va a seguir defendiendo la asimetría de las relaciones entre el centro Uruk y su periferia. Aun así, toma en consideración las críticas recibidas y formula un sistema-mundo de menor intensidad, marcado ahora por situaciones diversas, más acordes a la evidencia de los nuevos sitios hallados. Planteados los modelos teóricos, ya estamos en condiciones de proceder a revisar las evidencias principales de los sitios seleccionados. Pero, antes, es necesario describir brevemente la cultura Uruk, pues de esta manera podremos ponderar mejor el peso de su influencia en el proceso de urbanización en la Alta Mesopotamia.

\section{Cultura Uruk}

La cultura Uruk debe su nombre al yacimiento de Uruk-Warka, situado en la ribera oriental del Éufrates inferior, en la llanura aluvial mesopotámica, a 225 $\mathrm{km}$ de la actual ciudad de Bagdad. Las primeras excavaciones se llevaron a cabo desde mediados del siglo XIX y se han extendido bajo diferentes direcciones y con largos lapsos de interrupción hasta la actualidad. En las excavaciones realizadas en los años treinta se llevó a cabo un sondeo estratigráfico en el área del Eanna, a raíz del cual se propuso una datación dividida en tres fases: Uruk Antiguo (niveles XII a XI del Eanna) que iría del 4000 al 3800 a.C.; Uruk Medio (niveles X y IX del Eanna) del 3800 al 3600 a.C.; y Uruk Tardío (niveles VIII y IV del Eanna) del 3600 al 3200 a.C. (Nissen, 1988). 
La cultura Uruk se identifica a través de varios patrones: una arquitectura monumental de planta tripartita; paredes decoradas con conos de arcilla; cuencos de borde biselado, una cerámica tosca producida de manera estandarizada; bullae, es decir, esferas huecas de arcilla que contenían fichas o tokens; sellos cilíndricos, que junto a lo anterior denotaba una intensa actividad administrativa, como se desprende de su glíptica; y por último la escritura, utilizada por primera vez en unas tablillas pictográficas, encontradas en el nivel IVb del Eanna.

Respecto al fenómeno Uruk, Mario Liverani (2006 [1998]: 25-31) consideró que durante el Uruk Antiguo se produjeron innovaciones tecnológicas que permitieron un crecimiento exponencial de la producción agrícola. Los grandes trabajos hidráulicos para conformar canales de riego, así como el uso de arado y nuevos métodos de transporte de la producción, fueron el ejemplo más destacado de estos avances. La obtención de mayores excedentes en la producción agraria trajo consigo un incremento de la población en la zona. Durante el período Uruk Tardío, las prospecciones realizadas en el área demostraron el crecimiento exponencial del asentamiento, que llegaría a alcanzar las 250 hectáreas. Algaze (2004 [1993]: 193-196) concibió que el crecimiento de la economía generó la aparición de un comercio incipiente y de una élite dominante que entraría en competencia con las élites de otras ciudades cercanas. En el marco de esta competencia, o incluso sin ella, pero en el orden de obtener recursos de difícil acceso, para Algaze Uruk mantendría un rol central en las interacciones con regiones alejadas, avanzando hacia la meseta irania y los montes Zagros, los cauces superiores del Tigris y el Éufrates, el Levante mediterráneo, Anatolia y los montes Tauros.

Retomando lo ya expuesto, se puede inferir que el crecimiento urbano durante Uruk Tardío en la llanura aluvial estuvo caracterizado por los siguientes rasgos que planteó Liverani (2006 [1998]): el aumento de la estratificación social, visible a través de la glíptica y la iconografía del período; la aparición de una minoría dirigente político-religiosa, entendida tanto desde la arquitectura, que muestra un sentido de control administrativo y cultual, como de los cuencos de posible uso redistributivo; y por último, la existencia de una especialización laboral, producto de un floreciente comercio y evidenciada en los restos de bienes manufacturados de diverso valor.

\section{Urbanización local y expansión Uruk en la Alta Mesopotamia}

\section{Habuba Kabira}

El primer asentamiento al que nos referimos en este trabajo es Habuba Kabira, excavado intensivamente a fines de los años sesenta con motivo de la construcción de la represa de Taqba en el valle del Éufrates medio. El yacimiento estaba compuesto por dos sectores: la ciudad baja, Habuba Kabira Sur, y la acrópolis Tell Qannas, ambas conectadas por una amplia avenida (Akkermans y Schwartz, 2003: 190-194). El sitio de Habuba Kabira Sur es el más grande y mejor conocido de varios asentamientos hallados en 
la zona. Ocupando alrededor de 18 hectáreas, el sitio posee un área de 10 hectáreas amurallada y un montículo relacionado en el sudoeste. El yacimiento ha sido dividido en tres niveles de construcción. Sabemos gracias a esto y a la pequeña acumulación de restos hallados que su ocupación fue breve, extendiéndose alrededor de 200 años durante el período Uruk Tardío (CT 4 y 5, ca. 3400-3000 a.C.) para finalizar luego de forma abrupta. No se encontró evidencia de que el abandono del área haya sido motivado por sucesos vinculados a la violencia; sin embargo, la existencia de las murallas y su acelerado levantamiento permiten considerar relaciones beligerantes con los demás habitantes de la región.

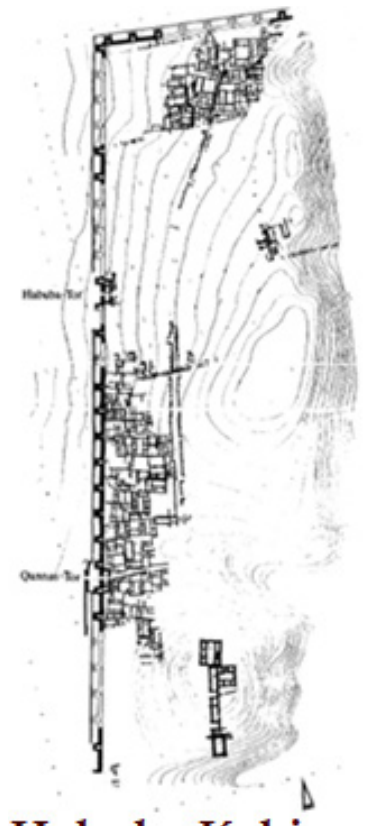

Habuba Kabira

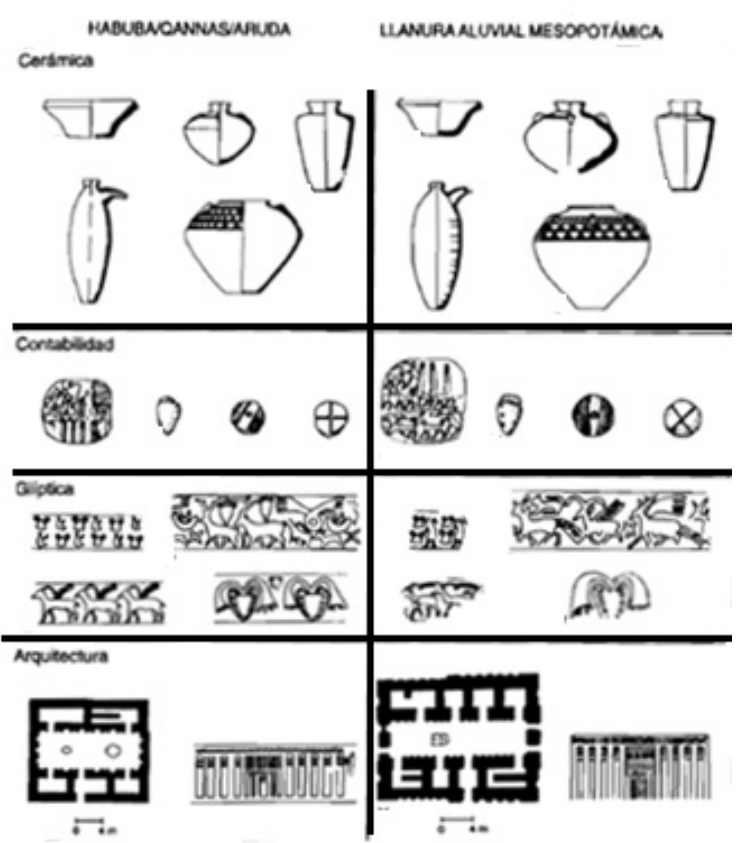

Sitio de excavación de Habuba Kabira (izq. adaptado de Akkermans y Schwartz, 2003: fig. 6.7) y paralelos en evidencia material entre Habuba Kabira y la Baja Mesopotamia (der. adaptado de Algaze, 2004 [1993]: fig. 17).

La arquitectura de Habuba Kabira exhibe un alto grado de uniformidad, con calles pavimentadas con gravilla ordenadas norte-sur y este-oeste así como un sofisticado sistema de drenaje, lo que plantea una posible planificación del asentamiento urbano antes de su construcción. Esto permite inferir la existencia de una administración de la comunidad, provista por una autoridad central llegada junto con los colonos a la zona. Respecto a las características arquitectónicas, el modelo tripartito de construcción fue usado repetidamente tanto para los edificios domésticos como para aquellos de uso público. En el sitio de Tell Qannas, las edificaciones muestran el estilo cultural característico del sur mesopotámico, con plantas tripartitas, nichos en las paredes, muros gruesos y conos de arcilla. La cerámica también manifiesta el estilo Uruk: abundan los cuencos de borde biselado, tanto completos como rotos. Los registros recuperados en las residencias excavadas presentan rasgos de administración burocrática exportados de la Baja Mesopotamia. También se encuentran en gran cantidad tablillas de arcilla que contienen símbolos numéricos y sellos cilíndricos (Kolhmeyer, 1996; Algaze, 2004 [1993]). 


\section{Hacinebi Tepe}

Hacinebi es un tell de 3,3 hectáreas que se encuentra en el sureste de Turquía, $5 \mathrm{~km}$ al norte de la moderna Birecik, en la provincia de Șanliurfa, en la orilla este del Éufrates y a los pies de los montes Tauros. En el área se puede realizar agricultura de secano gracias a una media de lluvias de entre $400 \mathrm{y}$ $600 \mathrm{~mm}$ anuales. La zona atraviesa una importante ruta de comunicación que, a través del Éufrates, une las minas de cobre del este de los Tauros, con Siria y más al sur con Mesopotamia (Stein, 2001: 268-270). La ocupación durante el IV milenio a.C. ha sido dividida en tres fases: una fase A (41003800 a.C.), con cerámicas locales del CT 2 (ca. 4200-3900 a.C.); una fase B1, entre el 3800 y 3700/3600 a.C., con formas tardías de cerámica local; y, por último, una fase B2, donde junto con la cerámica local se encontró otra de estilo meridional, evidencia del contacto y las interacciones con la Baja Mesopotamia.

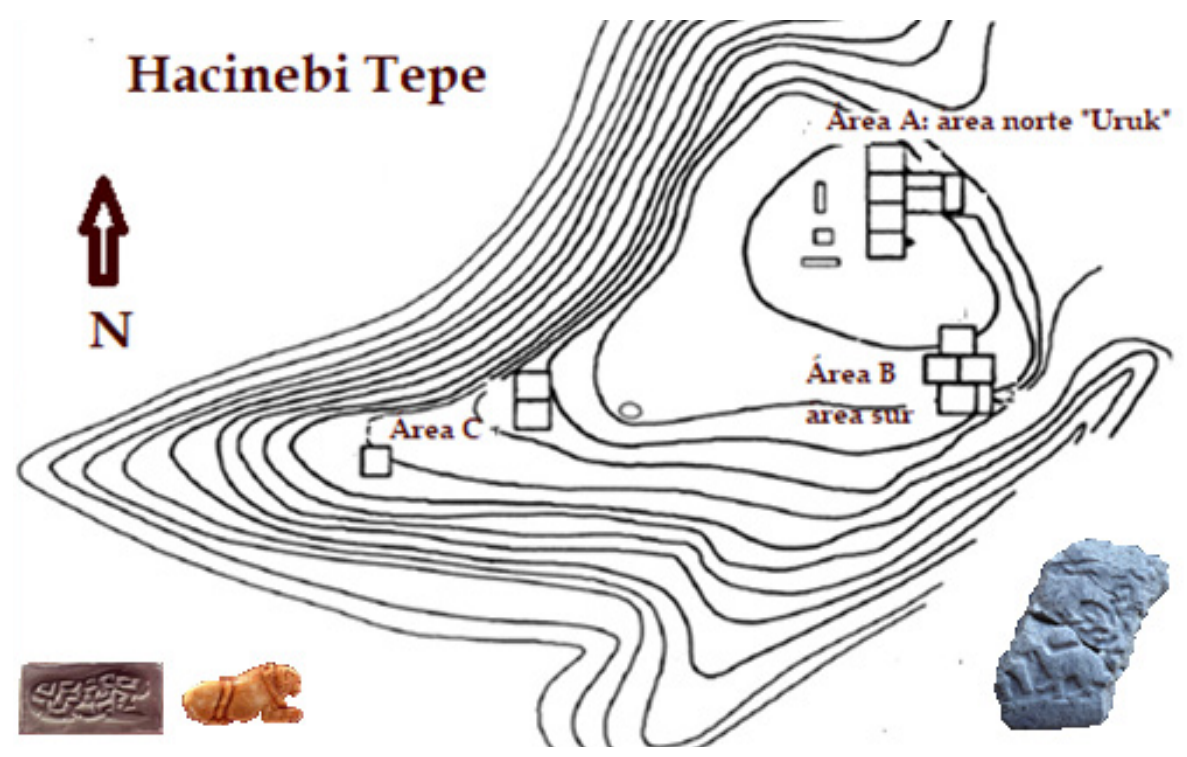

Plano del sitio de Hacinebi Tepe, en Turquía (adaptado de Stein, 2001: fig. 8.1).

Durante el período previo al contacto, encontramos arquitectura pública de gran escala en las tres áreas centrales de excavación. Especialmente notoria es una serie de depósitos de piedra relacionados con la metalurgia y actividades administrativas, así como un muro de piedra de más de tres metros de altura en el extremo sur del yacimiento. También se observan prácticas de enterramiento de tradición local -enterramientos de niños en tinajas de almacenamiento- y cerámica característica de la región. Por otra parte, la aparición de minerales foráneos en bienes de prestigio, evidencia la existencia de un comercio de larga distancia en el sureste de Anatolia. Ya durante la fase A está documentado en la glíptica el uso de sellos estampa como elemento administrativo que presenta paralelos, principalmente iconográficos, con los motivos hallados en los yacimientos de Tepe Gawra y Tell Brak (Stein, 2012: 136-138). Los motivos más utilizados involucran caprinos y felinos, y en menor medida serpientes. Las cretulae de esta región se utilizaron principalmente para sellar contenedores de almacenamiento 
y de transporte de bienes de comercio. Un elemento más a tener en cuenta cuando hablamos de una cultura local que es a la vez común a la Jezirah y a gran parte de Anatolia meridional es la presencia de "ídolos de ojos", estatuillas de caliza y otros materiales con ojos prominentes, documentadas tanto en Hacinebi como en Tepe Gawra, Arslantepe, Tell Hamoukar y Tell Brak (Stein, 2012: 141).

La fase B1 presenta una clara continuidad en las prácticas de enterramiento y los estilos cerámicos. Además, se observa la construcción de una plataforma elevada de piedra en el extremo noreste del sitio y la creación de un amplio espacio abierto hacia el este de la misma. Al no conservarse restos de la estructura original, sólo puede suponerse que el propósito de esta área podría tener fines religiosos o estar vinculado al control de una élite local (Stein, 2001).

En la fase B2, con mayor asiduidad desde mediados del IV milenio a.C., se observa una cultura material idéntica a la de la llanura meridional y a la de otros asentamientos del Éufrates medio. La aparición de cerámicas Uruk se concentra principalmente en el área noreste del sitio, donde se construyeron habitaciones de posible uso residencial. Aquí se han encontrado elementos administrativos tanto de origen local como foráneo. La reorganización arquitectónica del yacimiento y la aparición de esta nueva cultura permiten pensar en la creación de un enclave mesopotámico dentro del asentamiento en el sector noroeste, donde se concentra principalmente su cultura material. Mientras tanto, las zonas sur y oeste del sitio conservaron la predominancia de cultura material local. La cerámica Uruk hallada no es importada, fue realizada en el sitio y es contemporánea a la cerámica local. Asimismo, está atestiguado el uso de conos de arcilla en las paredes, rasgo decorativo típico de la Mesopotamia. Aparece de manera abundante también bitumen, una sustancia de uso mesopotámico y que posiblemente fue traída de la región del Hìt a orillas del Éufrates o de la planicie de Deh Luran (Stein, 2001: 288-289). Durante esta fase, se puede hablar de la existencia de dos sistemas administrativos diferentes en el área. Por un lado, un sistema local, existente desde principios del IV milenio a.C. y que ya describimos brevemente en el párrafo anterior. Por otra parte, un sistema caracterizado por el uso de cilindro sellos y bullae, comunes en Mesopotamia, pero prácticamente inexistentes en Anatolia.

\section{Tell Brak}

Tell Brak es un yacimiento en el noreste de la actual Siria, que se encuentra a unos $50 \mathrm{~km}$ de la ciudad de Al-Hasaka. En la primera mitad del IV milenio a.C. alcanzó las 43 hectáreas de extensión, pero a su alrededor también se documentaron toda una serie de tells, pequeñas aldeas, que podrían dar a entender una aglomeración urbana superior quizás a las 100 hectáreas. Estos sitios en la periferia cercana eran pequeños, el más grande no superaba las cinco hectáreas, lo que tal vez indicaría una dominación política y económica de Brak sobre sus comunidades satélite (Espejel Arroyo, 2014: 297-298). El sitio de Brak está localizado en el margen meridional del alto Khabur, en una región relativamente seca donde las precipitaciones rondan los $300 \mathrm{~mm}$ anuales. La importancia de Brak radica en contar con un terreno aprovechable 
para la agricultura, su posición estratégica como paso hacia las planicies del alto Khabur desde el sudeste, ser el punto de control de la región de Sinjar y, por último, su interacción fluvial directa con la Baja Mesopotamia. En este apartado abarcamos un recorte temporal que tomó desde el período $\mathrm{D}$ de Brak hasta el G. Este recorrido comprende la totalidad del Calcolítico Tardío, es decir, desde el 4400 hasta el 3000 a.C. aproximadamente (Emberling, 2002; McMahon et al., 2007).

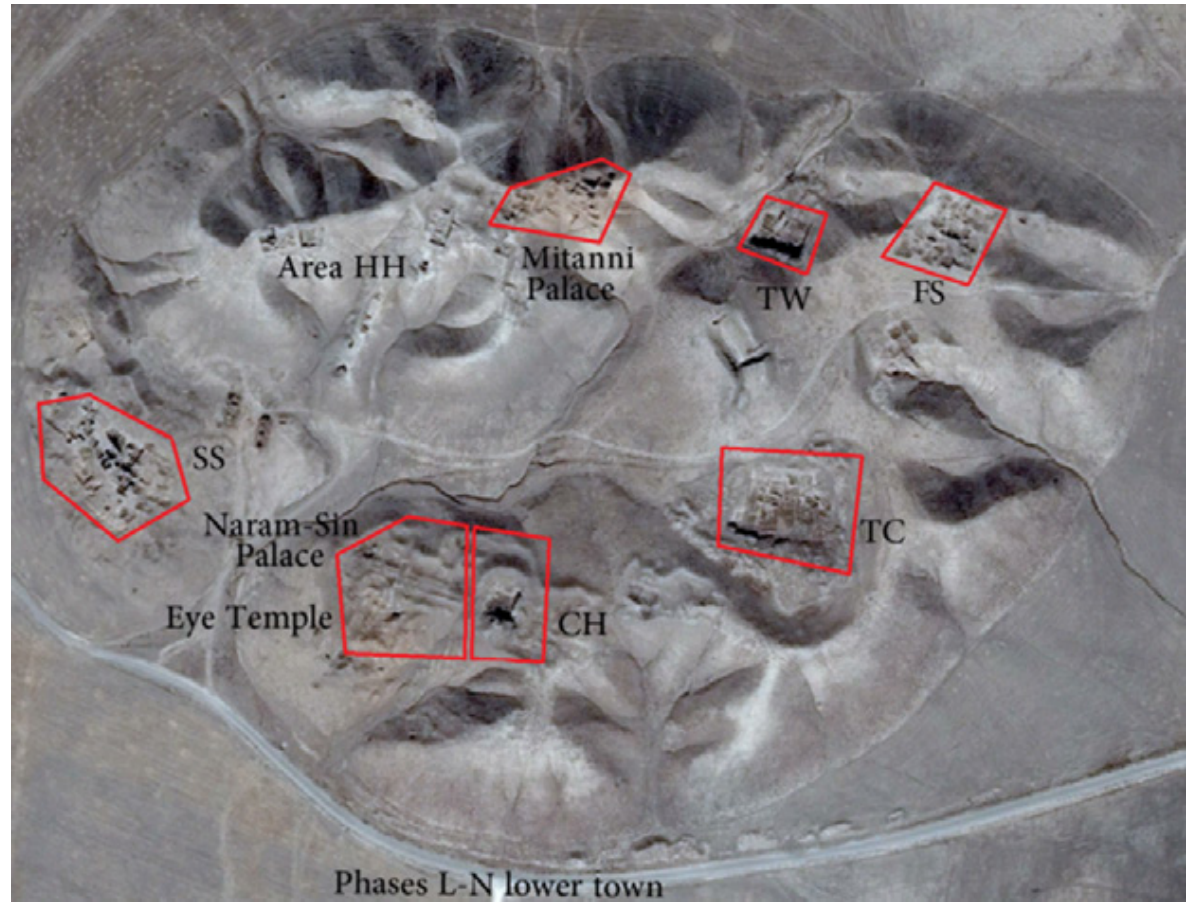

Yacimiento de Tell Brak (tomado de Wikimedia Commons, Attar-Aram, Syria).

El material hallado en el CT 1 (ca. 4400-4200 a.C.) corresponde a una fase postUbaid y es de origen local. Aquí aparecieron tempranamente cuencos coba, de producción en masa, con base plana y raspado en la parte inferior del cuerpo. Los primeros indicios del desarrollo de una mayor complejidad sociopolítica pueden verse a partir del CT 2 (ca. 4200-3900 a.C.). Durante este período, el asentamiento del tell se amplió exponencialmente, llegando a abarcar unas 30 hectáreas. Esta fase estuvo determinada por la continuación del uso de cuencos coba así como por la aparición de cerámica decorada con incisiones en zigzag y bruñido gris. También se han hallado restos de cerámica pintada sprigware. En el nivel 22 del área TW se encontraron grandes cantidades de obsidiana de procedencia diversa. En el plano arquitectónico, las excavaciones en los niveles TW 20-18 revelaron construcciones de uso público con muros de dos metros de grosor. Entre otras estructuras, encontramos el "edificio monumental" y el "edificio rojo". Así como construcciones que, por la gran cantidad de materiales y hornos hallados, permiten suponer un uso industrial. En este período también encontramos variada evidencia del uso de sellos. Las improntas de sello de los niveles 22 a 18 del área TW muestran nuevamente un estilo local y un interés creciente en la gestión del control y acceso a los bienes, ya que aparecen sobre contenedores trasladables y sobre puertas. 
El CT 3 (3900-3600 a.C.) demarcó la fase de mayor extensión del sitio, alrededor de 43 hectáreas, que, si se toman en cuenta los pequeños sitios cercanos, permite considerar una aglomeración urbana cercana a las 100 hectáreas. Está caracterizado por una cultura material local, entre la que aparecen bienes que denotan un alto estatus y complejidad organizativa como tablillas numéricas o marcas de alfarero en cerámicas. Durante este período comienzan a aparecer cuencos de borde biselado, cerámica propia de Uruk Medio, que se volvería abundante en el nivel TW 13. En los niveles posteriores, se encontraron grandes cantidades de improntas de sellos cilíndricos e incluso ciertos patrones arquitectónicos comunes a los sitios de Habuba Kabira y Jebel Aruda en el Éufrates medio (Akkermans y Schwartz, 2003: 198-200). No se puede dejar de nombrar ahora a la estructura monumental más conocida de Tell Brak, el "templo de los ojos". A lo largo de sus cuatro fases de ocupación se encontraron en sus recintos miles de ídolos oculados, figurillas de animales y sellos estampa, así como cilíndricos por igual. Ya desde su primera fase, del "templo rojo", se observa cerámica de englobe rojo y conos de arcilla, ambos característicos de la cultura Uruk. Durante la segunda fase; del "templo gris", aparecen cuencos de borde biselado, lo que permite datar esta fase en el CT 3 (Emberling, 2002: 84).

En este mismo período, se fecha el sitio de Tell Majnuna, un pequeño montículo a unos 500 metros de Tell Brak que presenta evidencia de cuatro enterramientos masivos de individuos jóvenes junto a cerámicas e improntas de sello. Los cuerpos estaban parcialmente desmembrados y presentaban lesiones traumáticas, lo que permite conjeturar un episodio de violencia entre poblaciones del triángulo del Khabur o quizás incluso el reflejo de una conquista Uruk, ya que el período se corresponde con el incremento de elementos de la cultura meridional en el área (Soltysiak, 2010). Aunque esta última posibilidad es discutible, profundizaremos en la hipótesis de la conquista de Tell Brak al final del trabajo.

Hacia el final del IV milenio a.C., en la fase TW 12 de Tell Brak, la cerámica era casi exclusivamente Uruk, análoga a la que encontramos en Habuba Kabira y otros yacimientos que son considerados como colonias de Uruk. Asimismo, en W 11-10 y Brak H, la cerámica del sitio incluye vasijas altas afines a las halladas en el período Jemdet Nasr en la Mesopotamia meridional. 


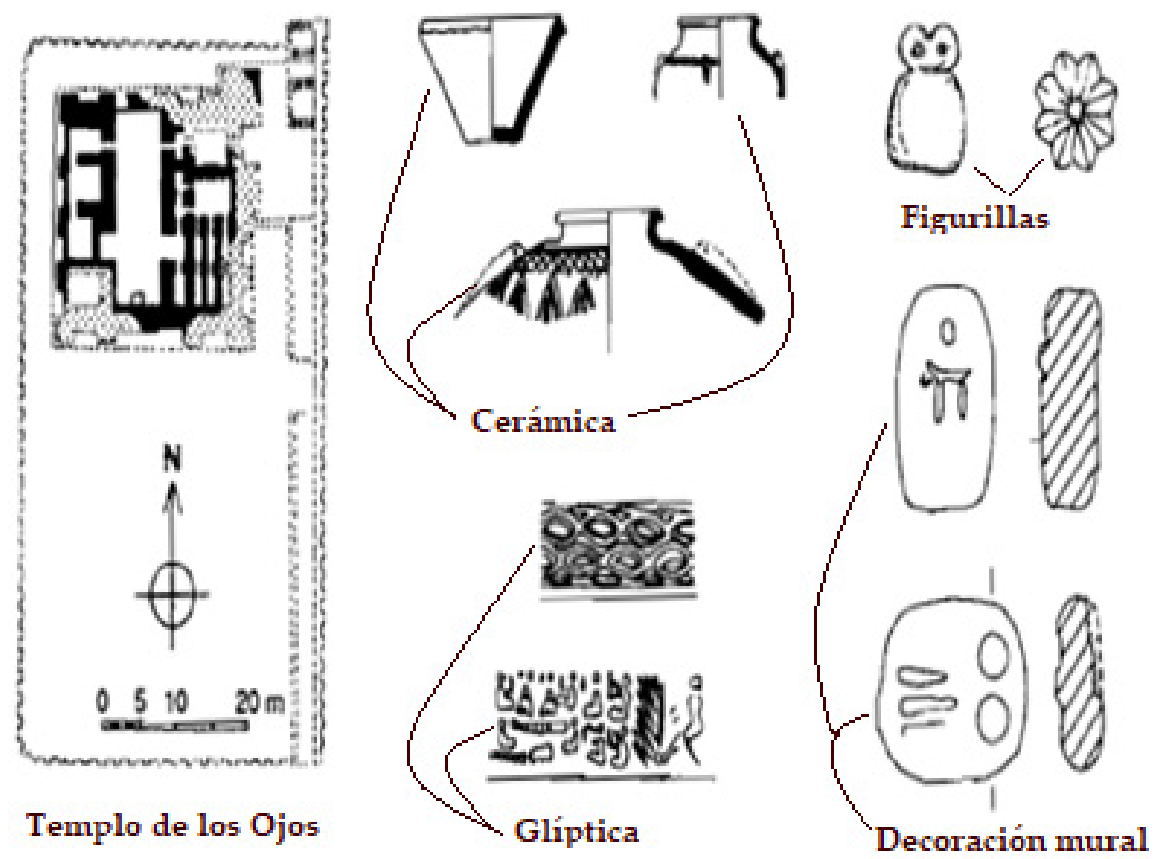

Elementos de cultura local y Uruk en Tell Brak (adaptado de Algaze, 2004 [1993]: fig. 18).

\section{Discusión}

Hemos observado que los debates sobre la urbanización y las relaciones en la macrorregión han gravitado sobre la aplicabilidad del sistema-mundo como marco teórico. La evidencia arqueológica ha servido para plantear la posibilidad de situaciones de mayor complejidad que las que se consideraron en un principio. A la luz de las recientes investigaciones, el modelo del sistema-mundo puede tomarse en cuenta, pero con reparos. El rol secundario del fenómeno urbano en el norte de Mesopotamia y su formación como producto directo de la influencia Uruk ha sido matizado y discutido por los hallazgos de los últimos años. En este sentido, ha quedado demostrada la existencia de comunidades complejas desarrollándose desde época Ubaid a través de la aparición de edificaciones monumentales previas al contacto con Uruk y que comparten rasgos comunes en todo el sureste de Anatolia y la Jezirah siria (Rothman, 2009: 18). Aun así, algunos de los supuestos del sistema-mundo han sido de gran utilidad para reinterpretar las relaciones en el Cercano Oriente en un plano de interacción interregional. A continuación, gracias a este marco teórico y a las características de los sitios estudiados en este trabajo, sugerimos la existencia de por lo menos tres formas de interacción, correspondientes a cada uno de estos sitios.

El caso de Habuba Kabira, por sus particularidades, fue en principio el más fácil de circunscribir. Consideramos que este sitio representa el ejemplo más notorio de un grupo de inmigrantes proveniente de Uruk o alguna otra ciudad de la Mesopotamia meridional que se establecieron en un área previamente desocupada y controlaron la zona y su hinterland. Si consideramos la posibilidad de 
contactos con poblaciones seminómadas, podemos pensar a esta comunidad como una diáspora dominante o una colonia directa. En este espacio, los "colonos" construyeron un asentamiento con motivos tanto de índole tanto política como económica. Llamativamente, en las zonas que estudiamos, este tipo de estructura social sólo aparece en la región del Éufrates medio. La organización del entramado urbano denota una planificación previa a su construcción, lo cual indicaría la intención de establecer una colonia en el área (Algaze, 2004 [1993]: 56-60). Se podría apreciar entonces una autoridad administrativa que proveyó los recursos necesarios para esta apropiación orgánica del terreno. La posibilidad de un asentamiento conformado por refugiados enfrentados con la administración de su ciudad natal también se presenta factible (Yoffee y Seri, 2019: 187-189) y por lo tanto no puede ser descartada. La evidencia arqueológica hallada en el sitio, como ya hemos visto, pertenece solamente a la cultura Uruk. Este dato es el que permite fundamentar la ocupación exclusiva del área deshabitada por un grupo étnico bajomesopotámico.

El porqué de la construcción de este asentamiento, así como de otros similares continúa siendo motivo de debate. Nos inclinamos a apoyar una explicación fundamentada en razones económicas. Si las élites del bajo Éufrates tuvieron la intención de controlar las rutas que comunicaban con Anatolia y sus metales, esta valoración cobra sentido. Se puede conjeturar que el posible control de las rutas de intercambio aseguraría la obtención de bienes de prestigio, lo que ayudaría a cimentar la dominación ideológica en la sociedad y el poder de las élites en un período de creciente estratificación social. Dentro de esta posibilidad, la creación de colonias brindaría cierta seguridad a los mercaderes frente a incursiones de las poblaciones indígenas locales, así como de otras ciudades vecinas. El abandono relativamente abrupto de las colonias un par de siglos luego de su edificación sería otro indicio de su dependencia frente al centro rector. La construcción de murallas protectoras alrededor de Habuba Kabira denota a su vez la existencia, o por lo menos la posibilidad, de relaciones conflictivas con los habitantes de la región. Aunque en este caso se puede observar una relación de centro-periferia, no debemos dejar de lado la fragilidad de la dominación de estos estados que se encuentran en un proceso de formación reciente. A eso debe sumarse la barrera natural que constituyen las distancias geográficas entre estos polos y que evoca el modelo de distance-parity.

El caso de Hacinebi Tepe es, en varios sentidos, distinto al de Habuba Kabira. La evidencia arqueológica permite reconstruir un sitio ya habitado por población de origen local con su propio acervo cultural, expresado a través de glíptica e iconografía diferente a la hallada en la Mesopotamia meridional. El uso de los sellos estampa y la arquitectura monumental son los indicios que demuestran la existencia de una comunidad compleja y estratificada con una administración desarrollada. Alrededor de 3700 a.C., se produjo la llegada de grupos mesopotámicos, posiblemente con intención de participar en el comercio de metales que ya existía en la zona (Stein, 2001: 295-293). Estos contactos estarían determinados por relaciones de intercambio y alianzas estratégicas con la élite local. Los estilos culturales anatólicos y mesopotámicos convivieron en Hacinebi durante al menos 400 años (Stein, 2012: 141-145). Las comunidades habitaron y se relacionaron entre sí desde dos zonas bien marcadas del asentamiento, y mantuvieron así sus propias identidades culturales. No hay nada 
que demuestre signos de imitación o transculturación. La comunidad local y su élite no emularon la cultura Uruk ni buscaron reemplazar su estilo cultural por el bajomesopotámico.

En Hacinebi, lo que podríamos ver es el establecimiento de un puesto avanzado de la sociedad Uruk. Sin embargo, no podemos presuponer un control demasiado fuerte de la urbe central sobre este puesto. La distancia y el nuevo grado de relaciones con la sociedad local pondrían en juego los lazos que unían al grupo inmigrante con su ciudad natal. De igual forma, la participación directa en la formación de esta comunidad autónoma por parte de Uruk es solamente conjeturable. No deja de ser interesante observar que la cultura Uruk aparece en la parte más elevada del tell, espacio que suele estar reservado para los sectores sociales más privilegiados. Las posibilidades en este sentido son múltiples, pero lo que podemos manifestar con seguridad es lo siguiente: el grupo extranjero era una comunidad que, debido principalmente a la distancia, habría perdido los lazos con la ciudad bajomesopotámica donde se originó. Es decir, una diáspora socialmente autónoma, débil por su reducido tamaño y que, sin importar el rol que tuviese en la zona, dependía de sus relaciones con la comunidad local para su supervivencia.

Por último, en Tell Brak hemos encontrado un escenario heterogéneo, que permite pensar distintas posibilidades a la hora de analizar el contacto del sitio con Uruk. Las excavaciones recientes en el área TW de Brak proveen evidencia de la interacción de larga duración en los niveles 13 a 11. Los materiales Uruk en el yacimiento fueron introducidos luego de una larga secuencia cultural indígena, lo que invitaría a pensar una situación parecida al caso de Hacinebi. Sin embargo, la complejidad del yacimiento de Brak también abre la puerta a otras situaciones factibles. A partir de TW 12 los artefactos encontrados y la organización arquitectónica parecen pertenecer al tipo Uruk Tardío. Algunos autores (Emberling, 2002) opinan que los elementos decorativos característicos del sur de Mesopotamia y el cambio de la planta tripartita en la última fase de remodelación del "templo de los ojos", implican que el control político del asentamiento se encontraba en manos de grupos pertenecientes a la llanura aluvial. Por la importancia social y estratégica que pareció tener el "templo de los ojos", su modificación sólo podría haberse llevado a cabo por aquellos que controlaban Tell Brak. Siguiendo esta lógica, el sitio de Tell Majnuna podría indicar un enfrentamiento violento entre las élites locales y grupos foráneos que culminaría con la conquista de la ciudad. Sin embargo, debido a que las excavaciones realizadas en Tell Brak sólo representan una pequeña porción del yacimiento ocupado, el grado de intrusión Uruk es difícil de determinar. Por lo tanto, no está claro si existió un enclave foráneo dentro de un sitio indígena o si grupos Uruk desplazaron a la comunidad preexistente en toda el área.

A nuestro modo de ver, es posible que la postura de Frangipane (2009) sea quizás la más apropiada para explicar estas particularidades. Según la autora, aunque la evidencia hallada permite pensar el asentamiento de población de la llanura aluvial en la zona, también pueden considerarse otros motivos para justificar la presencia de estos restos: el aumento en la intensidad de las relaciones con el mundo Uruk a partir del 3500 a.C., basado en un sustrato anterior 
de relaciones existentes entre el sur y el norte, marcaría la posibilidad de una emulación de técnicas de producción por parte de la población de Brak. Uruk sería un punto de referencia simbólico para las poblaciones de Siria y Anatolia. Las élites de Tell Brak en sus interacciones con la llanura aluvial asimilarían e imitarían rasgos ideológicos y organizativos de la Baja Mesopotamia. Esto puede explicar los cambios estructurales incluso en el "templo de los ojos" y la evidencia material hallada en el área. En cierto sentido, esta postura pareciera acercarse al modelo de aculturación que nombramos al principio, pero no consideramos que la autora estuviera afirmando aquí que las poblaciones de Brak hayan sido "inferiores", sirviendo sólo como receptáculo pasivo de una cultura más compleja, sino la posibilidad siempre viable de la apropiación consciente de ciertos símbolos culturales como reafirmación del poder de la élite y su distancia con su propia sociedad local.

\section{Conclusiones}

Hace medio siglo, la evidencia arqueológica disponible permitió teorizar sobre cierta superioridad de Uruk frente a estos asentamientos alejados, e incluso la posible dependencia política, económica y tecnológica de estos últimos. Sin embargo, los hallazgos recientes en las excavaciones, así como la discusión teórica sobre el tema han trazado nuevos modos de entender las relaciones interregionales. Consecuentemente, se ha matizado la corriente que establecía a Uruk como una cultura donante y con un rol de influencia central incluso en territorios lejanos. El uso de la teoría del sistema-mundo ya no puede aplicarse de forma indiscriminada. Tanto desde el plano espacial como el temporal, el rol de Uruk en su periferia debe entenderse desde la heterogeneidad de situaciones factibles.

Nuestro aporte en este sentido retoma el acervo teórico y la evidencia material para reflexionar sobre las formas de interacción transregional y su relación con el proceso de urbanización. Desde nuestro punto de vista, ninguno de estos modelos cubre la multiplicidad de relaciones posibles. Establecer un sistema cerrado, como ya hemos dicho, no condice con la variedad de situaciones que presenta el Calcolítico Tardío en la Alta Mesopotamia y el sur de Anatolia. Ilustramos con un cuadro las principales características de los modelos que hemos esbozado en este artículo. Para el caso de Habuba Kabira, es lógico considerar la posibilidad de una colonia regida por Uruk o de una diáspora dominante que no pudo prolongarse en el tiempo. En Hacinebi Tepe, el hallazgo de restos materiales de dos culturas diversas implica otra complejidad: ¿existe un puesto comercial Uruk que domina a la comunidad local? ¿O acaso una diáspora autónoma convive y debe aliarse por necesidad con la sociedad autóctona? Al día de hoy, ambas hipótesis son viables. Incluso, observando el amplio rango temporal, debemos considerar que las relaciones pueden haber mutado entre estas dos situaciones. Por último, el caso de Tell Brak también presenta un desafío. Para el período analizado específicamente y a partir de la evidencia arqueológica, no es descabellado teorizar sobre conflictos armados regionales que provocaran o impulsaran la penetración cultural Uruk. Aun así, resulta más factible considerarlo como 
un centro urbano indígena, que después se desarrolló en contacto directo con la Baja Mesopotamia, incorporando, a lo largo del tiempo, algunas características sureñas que terminarían reproduciendo las esferas más elevadas de la sociedad local.

\begin{tabular}{|l|l|l|l}
\hline Sitio & Habuba Kabira & Hacinebi Tepe & \multicolumn{1}{|c|}{ Tell Brak } \\
\hline $\begin{array}{l}\text { Período y fecha } \\
\text { aproximada }\end{array}$ & $\begin{array}{l}\text { Uruk Eanna V-IV } \\
(3400-3000 \text { a.C. }\end{array}$ & $\begin{array}{l}\text { Hacinebi B2 (3700- } \\
3200 \text { a.C.) }\end{array}$ & $\begin{array}{l}\text { Brak TW 13 - 12, } \\
\text { período F - G (3600- } \\
3000 \text { a.C.) }\end{array}$ \\
\hline Zona geográfica & $\begin{array}{l}\text { Llanura del Éufrates, } \\
\text { área de Taqba en el } \\
\text { norte de Siria. }\end{array}$ & $\begin{array}{l}\text { Ribera del alto } \\
\text { Éufrates, sudeste de } \\
\text { Turquía. }\end{array}$ & $\begin{array}{l}\text { Planicies del Alto } \\
\text { Khabur, noreste de } \\
\text { Siria. }\end{array}$ \\
\hline Descripción & $\begin{array}{l}\text { Asentamiento } \\
\text { fortificado de } \\
\text { población de sur } \\
\text { Mesopotamia en } \\
\text { terreno deshabitado. }\end{array}$ & $\begin{array}{l}\text { Pequeño enclave } \\
\text { mesopotámico en el } \\
\text { rincón noreste de un } \\
\text { asentamiento local } \\
\text { ya existente. }\end{array}$ & $\begin{array}{l}\text { Ocupación y pleno } \\
\text { desarrollo del sitio } \\
\text { antes de la aparición } \\
\text { de material Uruk. }\end{array}$ \\
\hline $\begin{array}{l}\text { Cultura } \\
\text { material y } \\
\text { restos hallados }\end{array}$ & $\begin{array}{l}\text { - Arquitectura } \\
\text { plenamente Uruk. } \\
\text { - Cerámica Uruk. } \\
\text { - Tablillas de arcilla } \\
\text { con registros } \\
\text { numéricos. } \\
\text { - Cilindros sello. }\end{array}$ & $\begin{array}{l}\text { - Arquitectura sur } \\
\text { mesopotámica en } \\
\text { espacio acotado. } \\
\text { - Bitumen. } \\
\text { - Cilindros sello. } \\
\text { - Arquitectura local y } \\
\text { sellos estampa. }\end{array}$ & $\begin{array}{l}\text { - Arquitectura con } \\
\text { influencia Uruk pero } \\
\text { con características } \\
\text { locales. } \\
\text { - Cuencos de borde } \\
\text { biselado. } \\
\text { - Ídolos de ojos, } \\
\text { influencia regional. }\end{array}$ \\
\hline $\begin{array}{l}\text { Modelos } \\
\text { factibles }\end{array}$ & $\begin{array}{l}\text { - Colonia Uruk. } \\
\text { - Diáspora } \\
\text { dominante. }\end{array}$ & $\begin{array}{l}\text { - Diáspora autónoma } \\
\text { junto a sociedad } \\
\text { local. } \\
\text { - Puesto comercial } \\
\text { Uruk en } \\
\text { asentamiento nativo. }\end{array}$ & $\begin{array}{l}\text { Centro indígena con } \\
\text { influencia Uruk de } \\
\text { intensidad variable. }\end{array}$ \\
\hline
\end{tabular}

Cuadro comparativo de yacimientos en el Cercano Oriente antiguo.

\section{Agradecimientos}

La preparación de este artículo comenzó durante el seminario de grado dictado por el Dr. Pablo Jaruf (UBA/CONICET) y la Lic. María Belén Daizo (UBA/ UNIPE): "La Revolución Urbana en Siria-Palestina: de las aldeas a las primeras ciudades" en 2018. Mi primer agradecimiento va para ellos por su valiosa guía y acompañamiento.

No puedo dejar de apreciar igualmente los comentarios y aportes de mis colegas del proyecto FILOCyT 19-075 "Ciclos de urbanización y dinámicas sociopolíticas en Siria-Palestina: de la Edad del Bronce a la Edad del Hierro (3600-700 a.C.)", así como también al Dr. Marcelo Campagno (UBA/CONICET) y a mis compañeros en la cátedra de Historia Antigua I (Oriente) "A" de la UBA que inspiraron mi interés en los temas abarcados y me brindaron el espacio para trabajar sobre los mismos. 


\section{Q Bibliografía}

» Adams, R. McC. y Nissen, H. J. (1972). The Uruk Countryside. Chicago: University of Chicago Press.

»Akkermans, P. M. M. G. y Schwartz, G. M. (2003). The Archaeology of Syria. From Complex Hunter-Gatherers to Early Urban Societies (16000-300 BC). Cambridge: Cambridge University Press.

"Algaze, G. (1989). The Uruk Expansion, Cross-cultural Exchange in Early Mesopotamian Civilization, en: Current Anthropology 30: 571-608.

»Algaze, G. (2004 [1993]). El sistema-mundo de Uruk. La expansión de la primera civilización mesopotámica. Barcelona: Bellaterra.

》Butterlin, P. (2003). Les Temps proto-urbaines de Mésopotamie: Contacts et acculturation à lépoque d'Uruk au Moyen-Orient. París: CNRS Éditions.

»Emberling, G. (2002). Political Control in an Early State: The Eye Temple and the Uruk Expansion in Northern Mesopotamia, en: al-Ghailani Werr, L., Curtis, J., Martin, H. P., McMahon, A., Oates, J. y Reade, J. (eds.), Of Pots and Plans. Papers on the Archaeology and History of Mesopotamia and Syria presented to David Oates in Honour of his 75th Birthday. Londres: NABU, 82-90.

»Espejel Arroyo, F. (2014). El impacto de la expansión Uruk en el sureste de Anatolia y norte de al Yazira: interacción e intercambio, en: del Cerro Linares, C., Milán Quiñones de León, M. S., Alonso Moreno, C. V., Elices Ocón, J., González Herrero, O., Mysłowska, A., Per Gimeno, L. y Viaá Gutiérrez, A. (eds.), Economías, comercio y relaciones internacionales en el mundo antiguo. Madrid: Fullcolor Printcolor, 295-317.

》Frangipane, M. (2009). Rise and Collapse of the Late Uruk Centers in Upper Mesopotamia and Eastern Anatolia, en: Szienze dell'antichità 15: 25-41.

» Frangipane, M. (2018). Different Trajectories in State Formation in Greater Mesopotamia: A View from Arslantepe (Turkey), en: Journal of Archaeological Research 26:3-63.

» Juliano, D. (1988). Aculturación, en: Aguirre Batzán, A. (ed.), Diccionario temático de Antropología. Barcelona: PPU, 17-23.

"Kohl, P. (1987). The Ancient Economy, Transferable Technologies and the Bronze Age World-System: A View from the Northeastern Frontier of the Ancient Near East, en: Rowlands, M., Larsen, M. y Kristiansen, K. (eds.), Centre and Periphery in the Ancient World. Cambridge: Cambridge University Press, 13-24.

» Kohlmeyer, K. (1996). Houses in Habuba Kabira-South. Spatial Organisation and Planning of Late Uruk Residential Architecture, en: Veenhof, K. R. (ed.), Houses and Households in Ancient Mesopotamia. The 4oth Rencontre Assyriologique Internationale. Leiden: Instituut voor het Nabije Oosten, 89-103.

》 Liverani, M. (2006 [1998]). Uruk. La primera ciudad. Barcelona: Bellaterra.

" McMahon, A., Oates, J., Al-Quntar, S., Charles, M., Colantoni, C., Hald, M. M., Karsgaard, P., Khalidi, L., Sołtysiak, A., Stone A. y Weber. J. (2007). Excavations at Tell Brak 2006-2007, en: Iraq 69: 145-171.

» Nissen, H. J. (1988). The Early History of the Ancient Near East. Chicago: University of Chicago Press. 
»Pittman, H. (2001). Mesopotamian Intraregional Relations Reflected through Glyptic Evidence in the Late Chalcolithic 1-5 Periods, en: Rothman, M. S. (ed.), Uruk, Mesopotamia and its Neighbors: Cross-Cultural Interactions in the Era of State Formation. Santa Fe: School of American Research Press, 403-444.

»Postgate, J. N. (2002). Artefacts of Complexity: Tracking the Uruk in the Near East. Warminster: Aris and Phillips.

»Rothman, M. S. (2009). Religion, Function, and Social Networks: Tepe Gawra in the Late Fifth and Early Fourth Millennia BC, en: Butterlin, P. (ed.), A Propos de Tepe Gawra. Le Monde Proto Urbain de Mésopotamie. Subartu 23. Turnhout: Brepols, 15-39.

"Schwartz, G. M. (1988). Excavation at Karatut Mevkii and Perspectives on the Uruk/Jemdet Nasr Expansion, en: Akkadica 56: 1-41.

»Schwartz, G. M. (2001). Syria and the Uruk Expansion, en: Rothman, M. S. (ed.), Uruk, Mesopotamia and its Neighbors: Cross-Cultural Interactions in the Era of State Formation. Santa Fe: School of American Research Press, 233-264.

»Schwartz, G. M. y Hollander, D. (2008). Annealing, Distilling, Reheating and Recycling: Bitumen Processing in the Ancient Near East, en: Paléorient 26: 8391.

"Soltysiak, A. (2010). Death and Decay at the Dawn of the City: Interpretation of Human Bone Deposits at Tell Majnuna, Areas MTW, EM and EMS. Varsovia: Institute of Archaeology, University of Warsaw.

»Stein, G. J. (1990). Comments on G. Algaze: The UrukExpansion: Cross-Cultural Exchange in Early Mesopotamian Civilization, en: Current Anthropology 31: 6667.

»Stein, G. J. (1999). Rethinking World Systems: Diasporas, Colonies, and Interaction in Uruk Mesopotamia. Tucson: University of Arizona Press.

»Stein, G. J. (2001). Indigenous Social Complexity at Hacinebi (Turkey) and the Organization of Uruk Colonial Contact, en: Rothman, M. S. (ed.), Uruk, Mesopotamia and its Neighbors: Cross-Cultural Interactions in the Era of State Formation. Santa Fe: School of American Research Press, 265-305.

»Stein, G. J. (2002). From Passive Periphery to Active Agents: Emerging Perspectives in the Archaeology of Interregional Interaction, en: American Anthropologist 104 (3): 903-916.

"Stein, G. J. (2012). The development of indigenous social complexity in the Late Chalcolithic Upper Mesopotamia in the sth-4th millennia B. C. - An initial assessment, en: Origini 34: 125-151.

"Wallerstein, I. (1974). The Modern World-System I: Capitalist, Agriculture and the Origins of the European World-Economy in the Sixteenth Century. Nueva York: Academic Press.

»Yoffee, N. y Seri, A. (2019). Negotiating Fragility in Ancient Mesopotamia: Arenas of Contestation and Institutions of Resistance, en: Yoffee, N. (ed.), The Evolution of Fragility: Setting the Terms. Cambridge: McDonald Institute for Archaeological Research, 183-194. 